\title{
The first planets discovered from space with the CoRoT mission $\dagger$
}

\author{
Pierre Barge and the CoRoT Exoplanet Science Team \\ Laboratoire d'Astrophysique de Marseille - LAM/OAMP - UMR6110, CNRS/Université de \\ Provence - 38 Rue F. Joliot-Curie - 13388 - Marseille Cedex \\ email: pierre.barge@oamp.fr
}

\begin{abstract}
The pioneer space mission for photometric planet searches CoRoT continuously monitors about 12,000 stars in each of its fields of view. Thanks to the so-called "alarm mode", transit candidates can be detected early in the processing of the data and before the end of a run of observation. This specificity offers the possibility of rapidly triggering follow-up operations for the confirmation and characterization of the best transit candidates. We present the first objects discovered in this way: four planets and a transition object between brown dwarf and planet. We describe the organization of the CoRoT Exoplanet Science Team and briefly comment on the set of periodic signals identified by the various detection groups in the first three runs of observation.
\end{abstract}

\section{Introduction}

Led by the CNES space agency, CoRoT is performing the first space survey dedicated to a photometric search for extrasolar planets. The instrument and the mission are described in the previous chapter; a complete description can be found in a pre-launch book (CoRoT 2006) and in a post-launch paper (Auvergne et al. 2008). On the two CCDs devoted to extrasolar planet search, the measure of the stellar flux is performed every $512 \mathrm{~s}$, by piling up on board 16 individual exposures of $32 \mathrm{~s}$ obtained by aperture photometry. The instrument satisfies the initial scientific requirements of the mission with a noise level that matches the photon noise over most of the magnitude range accessible to CoRoT $(12-16)$.

The photometric apertures ("masks") on the CCDs are uploaded at the beginning of a run, after being selected from a pre-defined list of patterns. In addition to the target, a mask may contain contaminating stars that are potential sources of noise and transit false positives. Reference windows with a square shape are also selected in the non-exposed parts of the CCDs $(5 \times 5$ pixels $)$ for the offset corrections or in the darkest regions of the CCDs $(10 \times 10$ pixels $)$ for sky-background corrections.

In polar Earth orbit, CoRoT is free of the main limitations of ground-based transit searches. The photometric accuracy is better than $1 \mathrm{mmag}$, and the monitoring of the targets is nearly continuous over several months. However, the total duration of a run cannot exceed 6 months to avoid blinding by the Sun and occultation by the Earth. Another inconvenience comes from charged particle impacts at the crossing of the SouthAtlantic magnetic anomaly, as they can produce bright pixels on the CCDs and outliers in the lightcurves.

$\dagger$ The CoRoT space mission, launched on Dec. 27th, 2006, was developed and is operated by the CNES, with participation of the Science Program of ESA, ESTEC/RSSD, Austria, Belgium, Brazil, Germany and Spain. 
This paper reports the first discoveries issued from the alarm mode and comments on the periodic signals detected in the data by the various groups that are part of the CoRoT Exoplanet Science Team (CEST).

\section{2. "Alarm mode" and the CEST strategy}

One specificity of the CoRoT exoplanet program is the "alarm mode", an operational loop between science team and command center on the ground and the instrument in space (Quentin et al. 2006). Its goal is to optimize the science return of the mission by identifying early in the process, before the end of a run, transit candidates before the full data reduction. For these transit candidates, decision can be made to change the rate of the observations from one exposure in 512s to one exposure in 32s. The interest for this oversampling is two-fold: (i) to get a better coverage of the transit profile (in view of measuring stellar limb darkening and planetary albedo); (ii) to reduce the level of noise by removing outliers from the corrupted exposures. Last but not least, another interest of this "real-time" detection is to start follow-up operations as soon as possible.

Once transit signals are detected, the goal is to secure the detections and to trigger the follow-up observations necessary to completely characterize the discovered planets. Inside the CEST, the work necessary to reach this goal consists in 5 different tasks.

- Task 1: The first task, in direct relation with the detection procedures, is to make a detailed analysis of the light-curve and remove the possible false positives, for example by identifying indirect signatures of eclipsing binaries (secondary transit, triangular shape of the primary transit, ellipsoidal modulations due to tidal effects).

- Task 2: It consists in using in-transit/out-of-transit photometry to test: (i) the level of variability of the background stars identified inside the target PSF (Point Spread Function), (ii) whether one of the stars in the target PSF could mimic a transit occurring on the target. The necessary information on the contaminating stars is found in the ExoDat preparatory database that contains all the necessary information about the field of view and the targets.

- Task 3: Once the transit candidate is secured, radial velocities are used to confirm the presence of a planet and to measure its mass. If accurate enough, radial velocity measurements can also be used to reveal the Rossiter-Mac-Laughlin effect that put constraints on the alignment of the stellar spin and planetary orbit axes. Most of the CoRoT planet candidates will be within the reach of the SOPHIE and HARPS spectrographs.

- Task 4: It consists in obtaining precise stellar parameters using high-resolution spectroscopy and to get a good estimate of the planet parameters.

- Task 5: Finally, the last task is to use space observations, like HST, FIRST, etc... for in-depth studies and better characterisation of the planet parameters.

\section{Variable stars and eclipsing binaries}

From a quick inspection of the CoRoT light curves, the most striking aspect is the large number of stars with a significant level of variability. Some $40 \%$ of the targets in the exo-fields are identified as variables stars that can be periodic pulsators with periods 
from days to months. These stars are now sorted following a classification that must still be improved (Debosscher et al. 2007).

Eclipsing binaries are also frequently identified in the CoRoT field of view. They can be loose or tight systems with amplitudes that can be very large (several percents or more). A number of them show clear evidence of ellipsoidal variations due to tidal interactions between the two components. An example of eclipsing binaries found by CoRoT is given in Fig. 1, with a succession of primary and secondary eclipses. This example also illustrates the possible confusion between eclipsing binaries and transiting planets when the amplitude of primary and secondary eclipses are nearly equal and/or when the noise rubs out the difference between the two types of eclipses.

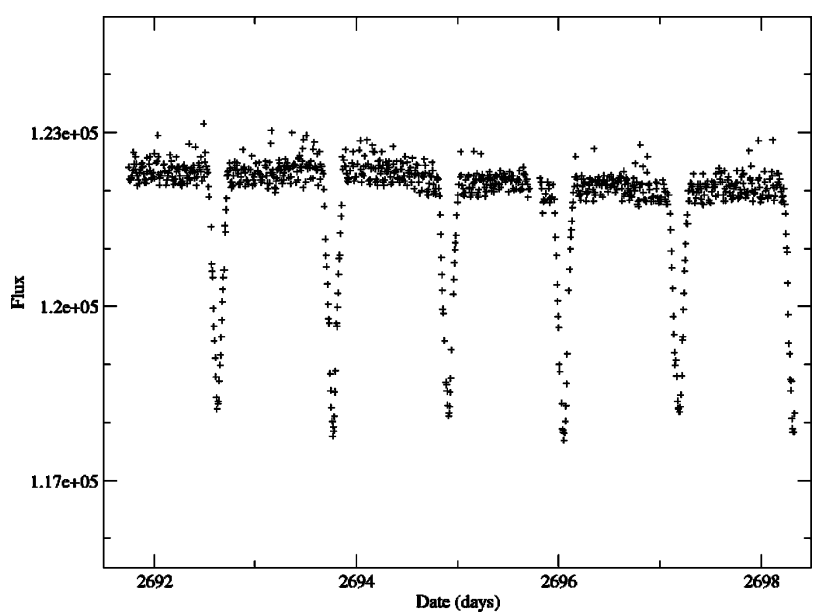

Figure 1. Example of eclipsing binaries in the CoRoT exofield.

The high frequency of eclipsing binaries in the CoRoT field of view and the large extension of the CoRoT PSF onto the sky (about 30 arcsec) are also the cause of possible confusions between the periodic eclipses of a binary star in the background and the transits of a planet in front of the target star. A number of ambiguous situations can be removed thanks to follow-up observations: either on/off photometry of contaminating stars in the photometric mask or Doppler spectroscopy of the target.

\section{The first planets detected from space}

In order to illustrate how the data are processed, the transits fitted and the planets characterized, we briefly describe the procedures that were used in the case of CoRoT-Exo-1 b (Barge et al. 2008). Data used for detection in the "alarm" mode are raw data that require consequent processing before yielding correct estimates of the transit parameters, particularly the transit depth. The necessary treatment of the data focuses on: (i) the background and offset corrections, (ii) the filtering of the outliers and lowfrequency residuals produced by crossing the South Atlantic Anomaly. These corrections are now implemented in the reduction pipeline that produces the N1 level data and also the completely reduced N2 data. In a second step, the light curve is successively (i) normalized with the median value at each orbit, (ii) phase-folded at the period of the satellite, and (iii) high-frequency filtered (using for example a $128 \times 128$ points 
Savitzky-Golay-weighted moving average filter, with 4th degree weighting). The detrended light curve is phase-folded, avoiding the few transits strongly affected by a bright pixel ( 2 transits out of 36 in the case of CoRoT-Exo-1b) and fitted with a model (using for example the formalism of Giménez, 2006).

In the case of CoRoT-Exo-1, the follow up procedure began with a first look at the archive of the pre-launch photometric survey performed with the Berlin Exoplanet Search Telescope (BEST). The target was indeed observed by BEST. However the data were too noisy to clearly identify a transit egress. Follow-up photometry with the Wise observatory $1.0 \mathrm{~m}$ telescope confirmed that a transit occurs on the main target. Images taken at the CFHT showed only some faint background stars $(\mathrm{V}>22)$ that confirm the weak contamination inside the CoRoT photometric aperture. The results of these investigations indicated that none of CoRoT-Exo-1's near neighbours can be responsible for the transit signal. High-precision, radial velocity observations of CoRoT-Exo-1 were made at Observatoire de Haute Provence in March-April and October 2007 with SOPHIE, an echelle cross-dispersed fiber-fed spectrograph at the 193-cm telescope (Bouchy et al. 2006). The radial-velocity curve supports the planetary nature of the transiting body detected by CoRoT and discards other interpretations involving background stars, grazing eclipsing binary, or a triple system.

Besides CoRoT-Exo-1b, three other planets were discovered using similar procedures for processing the data and characterizing the planets (see Fig. 2).
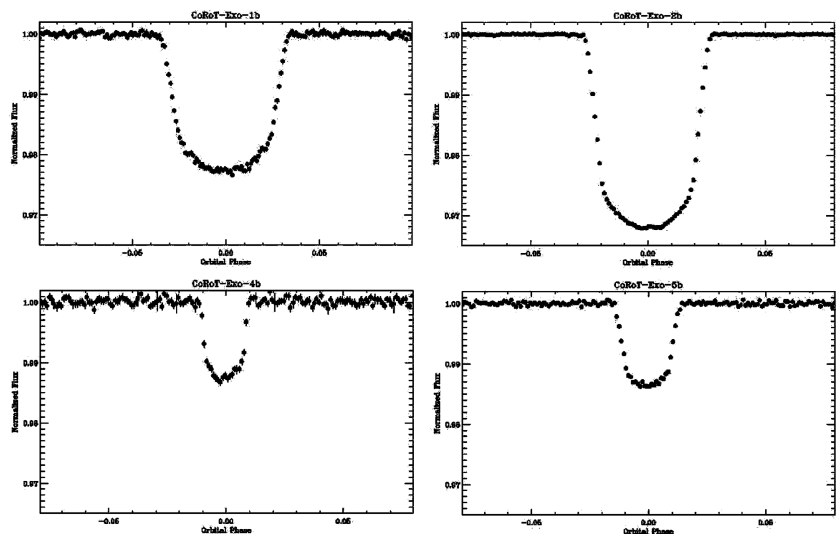

Figure 2. Phase-folded light curves of the first planets discovered from space by CoRoT. The number of transits is 36 for exo- $1 \mathrm{~b}, 81$ for exo- $2 \mathrm{~b}, 6$ for exo- $4 \mathrm{~b}$ and 27 for exo- $5 \mathrm{~b}$.

- CoRoT-Exo-2b was discovered around an active G type star (Alonso et al. 2008) Due to its high variability level the star would have been excluded from the standard radial velocity surveys and the planet would have been missed. The light curve is a very good example of the interest of long duration photometric surveys to search for extrasolar planets but also to sample the stellar variability. This light curve is also the first one that contains so many successive transits (86) in a single run.

- CoRoT-Exo-4b has a 9.2-day orbital period, the longest one among known transiting planets after HD 17156b (Barbieri et al. 2007) The orbit of the planet and the rotation of the envelope of the star seem to be synchronized even if the two periods are not exactly the same (Aigrain et al. 2008) 
- CoRoT-Exo-5b is a standard hot jupiter with a 4-day period (Rauer et al., in preparation).

- CoRoT-Exo-3b, orbiting a GV star, has the radius of a planet like Saturn but the mass of a sub-stellar object of 20 Jupiter masses. It could be a transiting brown dwarf whose radius can be measured for the first time, but also a transition object between a star and a planet, with important consequences for planetary formation models.

\section{A quick analysis of the three first runs}

The data of the three first runs of observation (IRa01, LRc01, SRc01) were reduced with a first validated version of the pipeline and distributed among the Co-Investigators. They correspond to some 30,000 light-curves that the detection groups have scrutinized looking for periodic signals and possible planetary transits. The various groups found in these light curves: (i) clear signatures of eclipsing binaries, (ii) transit candidates, (iii) "false positives" that can be due either to the confusion between the eclipses of a star and the transits of a planet on the target, or to background eclipsing binaries, or to residual instrumental noises.

On average, for each run of observation, we found in the data: (i) 30-40 transit candidates that are discussed case by case before selection for follow-up operations; (ii) $\sim 200$ eclipsing binaries and spurious detections (background eclipsing binaries or red noise). All the detected signals are displayed in plots (see Fig. 3-5) that give an idea of the detection capability of CoRoT. The transit depth is plotted as a function of the period of the event in Fig. 3, while Fig. 4 shows the transit period as a function of $R$-band magnitude.

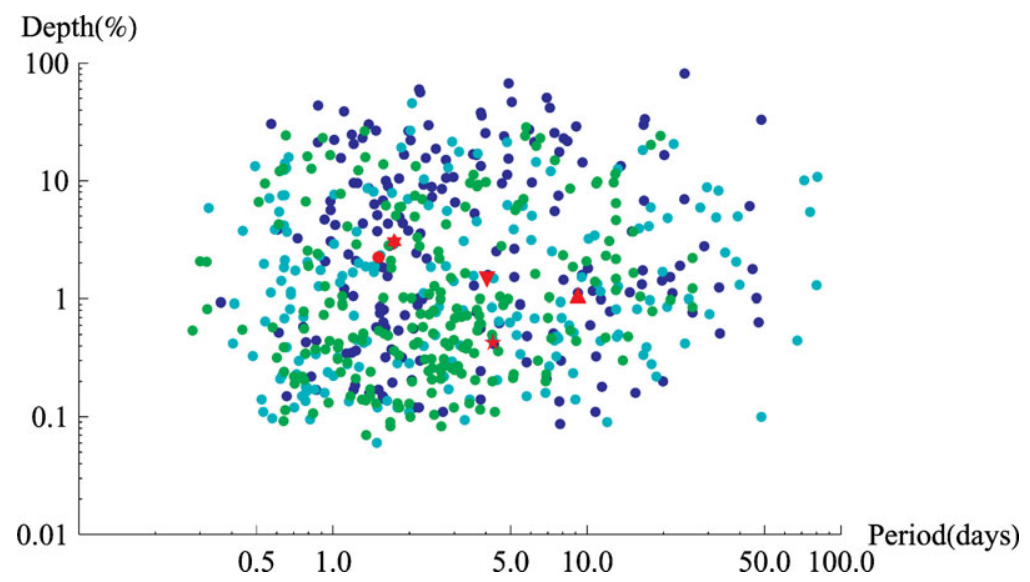

Figure 3. Depth versus period for the signals detected in the three first runs of observation. This plot is only a rough estimate of the CoRoT detection capability - (see text). Dark-blue dots correspond to detections in IRa01, light-blue dots to detections in LRc01 and green dots to detections in SRc01. In red are the confirmed planets (round-dot for exo-1b, 6-branch star for Exo-2b, 5-branch star for exo-3b, upward-triangle for Exo-4b, downward-triangle for Exo-5b).

It must be stressed that these two plots are very preliminary and must be examined with caution. Indeed, (i) results from eight different detection teams have been put in common, teams that use different methods for the detrending, the detection and the 
estimation of transit parameters, (ii) the data used for the detection were processed with the first version of the pipeline (an improved version will be soon available), (iii) the transit depth measured by the detection teams do not account for the contamination by the background and the nearby stars.

In any case, Fig. 3 shows two interesting features: (1) a pile-up of detections at the short-period end (this is consistent with the fact that the shorter the period, the easier the detection, but also with the pile-up of tight eclipsing binaries in the stellar background), (2) a "cut off" for transit smaller than about $0.1 \%$, that could indicate the limitation of the first version of the reduction pipeline and the difficulties of the detection teams to work with a high amount of bright pixels (see Baglin et al. in this volume).

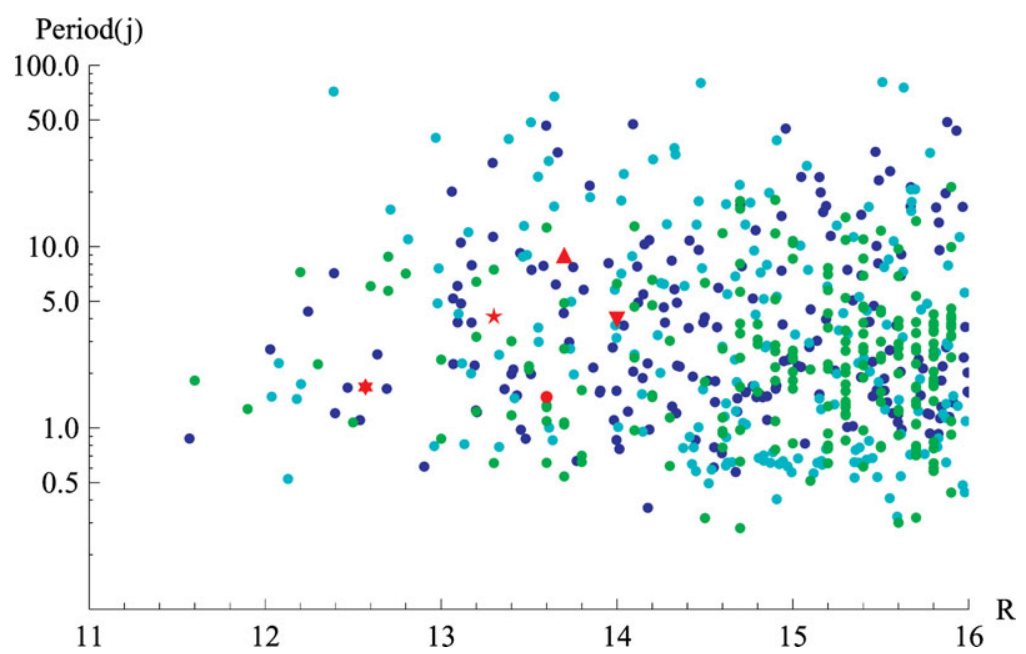

Figure 4. Transit period versus Red magnitude. The various symbols have the same meaning as in Figure 3.

When plotted as a function of magnitude, the results of the detection teams reveal the (expected) increasing number of events as a function of the magnitude of the stars. Note also the tendency to have a larger number of false positives for fainters stars (that is however detection-method dependant).

In fact, it must be stressed that: (i) very small transit signals $(\sim 0.05 \%)$ were found in alarm-mode data in the run LRa01 (but not likely to be due to planets), (ii) procedures are presently developed within the CEST to limit the consequences of the bright pixels on the detection efficiency, (iii) an improved version of the reduction pipeline will be implemented in a few months, (iv) detection teams will further improve their algorithms. The second interesting parameter is the transit duration, whose estimate may significantly differ from one team to another. The orbital period vs. transit duration plot for the three runs of observation (see Fig. 5) clearly show the correlation with a power law of index $1 / 3$, consistent with the relation between the duration and the period of the transits required for Keplerian orbits. Some biases in the upper part of the plot result from the fact that a number of detection team tend to remove obvious binaries from their results. 


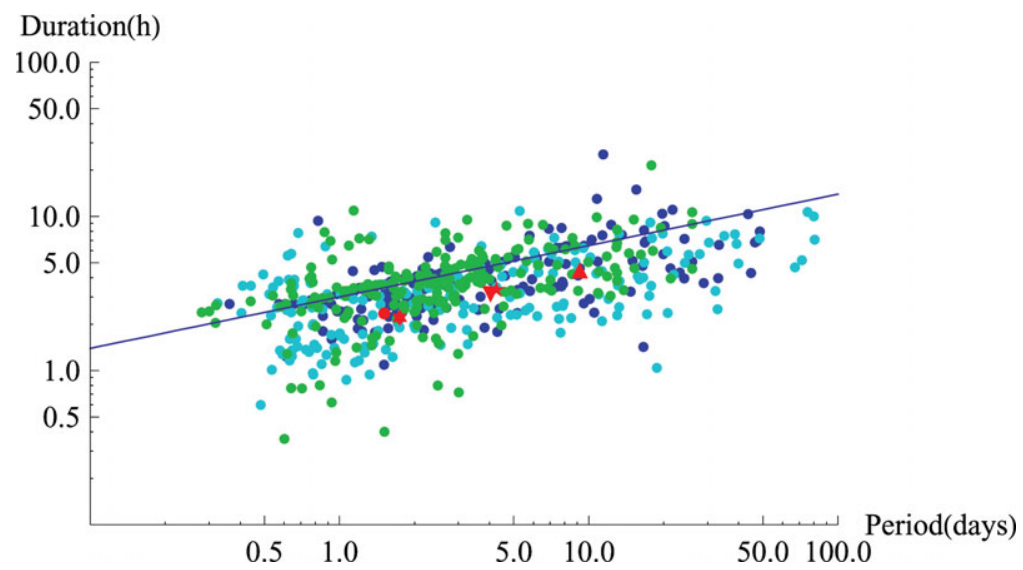

Figure 5. Transit duration versus period for the three first runs observed by CoRoT. The correlation (power law with index $1 / 3$ indicated by a full line) originates in the classical Kepler relation between transit duration and orbital period. The various symbols have the same meaning as in Fig. 3.

\section{Conclusions}

CoRoT is the first instrument to find extrasolar planets from space. The planets are completely characterized thanks to a ground-based follow-up strategy that can be triggered early in the processing of the data. Out of the first $\sim 30,000$ light curves, CoRoT has found 4 planets and what is probably the first completely characterized brown dwarf. More planets should be found once the data will be reduced with an improved version of the pipeline (better pointing jitter corrections) and a strategy to remove the bright pixels. For the time being, a blind test performed within the detection groups of the CEST (based on simulated transits within a sample of real light curves) seems to confirm that Uranus-size planets are within the reach of CoRoT.

\section{References}

Aigrain, S., et al. 2008, Astron. ES Astrophys.

Alonso, R., et al. 2008, Astron. \& Astrophys., 418 L21.

Auvergne, M., et al. 2008, Astron. \& Astrophys., submitted.

Barbieri, M., et al. 2007, Astron. \& Astrophys., 476, L13.

Barge, P., et al. 2008, Astron. \& Astrophys., 418, L17.

Bouchy, F. et al., 2006, in Tenth Anniversary of 51 Peg-b, 319.

CoRoT 2006, The CoRoT Mission: pre-launch status ESA-SP 1306, eds M. Fridlund, A. Baglin, L. Conroy \& J. Lochard.

Debosscher, J., Sarro, L. M., Aerts, C., et al. 2007, Astron. \& Astrophys. 475, 1159.

Deleuil, M., et al. 2008, Astron. \& Astrophys., (submitted).

Giménez A. 2006, Astron. ES Astrophys., 450, 1231

Rauer, H. et al. 2008, Astron. \& Astrophys., (in preparation).

Moutou, C., Bruntt, H., Guillot, T., Shporer, A. et al. 2008, Astron. ES Astrophys., (submitted). 